\title{
Utility of Neutrophil to Lymphocyte Ratio as a Predictor of Complications in Patients with Liver Cirrhosis
}

\author{
Vineeth V. K. ${ }^{1}$, Adithi Kellarai' ${ }^{2}$, Prakash P. S. ${ }^{3}$ \\ ${ }^{1}$ Department of General Medicine, Christian Medical College, Vellore, Tamilnadu, India. ${ }^{2}$ Department of \\ General Medicine, K.S. Hegde Medical Academy, Mangalore, Karnataka, India. ${ }^{3}$ Department of General \\ Medicine, K.S. Hegde Medical Academy, Mangalore, Karnataka, India.
}

\section{ABSTRACT}

\section{BACKGROUND}

Neutrophil to Lymphocyte Ratio (NLR) has been considered an inexpensive biomarker to reflect inflammation in patients with cirrhosis. Raised NLR has been associated with poor clinical outcome and mortality in these patients. We conducted this study to correlate the association of Neutrophil to Lymphocyte Ratio with complications of cirrhosis and assess the short-term outcome during hospital admission.

\section{METHODS}

We conducted a prospective observational study in 120 patients with cirrhosis from January 2017 to June 2018. All patients were diagnosed based on clinical history, examination and ultrasound. Complications were diagnosed by clinical and laboratory evaluation. Total white blood cell count, lymphocyte count, and neutrophil count were recorded, and neutrophil to lymphocyte ratio was calculated. Statistical analysis by means of test of association and binary logistic regression analysis was carried out.

\section{RESULTS}

Out of the 120 patients enrolled in our study, majority were male $(90 \%)$, and in the age group 51 - 60 years (38.3\%). Majority (39.2\%) of the patients had portal hypertension with oesophageal variceal bleeding as complication. Mean NLR for the patients was 5.824. A total of $91 \%$ patients with $\mathrm{NLR}>12$ were admitted with decompensated liver disease and $66.7 \%$ of those patients presented with more than 2 complications that is hepatic encephalopathy and spontaneous bacterial peritonitis. $41.7 \%$ patients with NLR $>12$ had mortality as the outcome. Chi square test of association of NLR with complications $(p=0.003)$ and mortality $(p=0.03)$ were significant. However, NLR could not predict complications in our study population.

\section{CONCLUSIONS}

Raised Neutrophil to Lymphocyte Ratio is associated with complication and mortality in patients with cirrhosis. However, it could not effectively predict the complications in patients with cirrhosis.

\section{KEY WORDS}

Biomarker, Cirrhosis, Complications, Decompensation, Neutrophil to Lymphocyte Ratio, Mortality

\author{
Corresponding Author: \\ Dr. Adithi Kellarai, \\ Assistant Professor, \\ Department of General Medicine, \\ K.S. Hegde Medial Academy, \\ Nithyanandanagar, Deralakatte, \\ Mangalore-575018, Karnataka, India. \\ E-mail: adithibhandary@yahoo.com
}

DOI: $10.14260 /$ jemds/2020/478

How to Cite This Article:

Vineeth VK, Kellarai A, Prakash PS. Utility of neutrophil to lymphocyte ratio as a predictor of complications in patients with liver cirrhosis. J. Evolution Med. Dent. Sci. 2020;9(31):2197-2201, $10.14260 /$ jemds/2020/478 DOI:

Submission 01-05-2020,

Peer Review 25-06-2020,

Acceptance 03-07-2020,

Published 03-08-2020.

Copyright (C) 2020 JEMDS. This is an open access article distributed under Creative Commons Attribution License [Attribution 4.0 International (CC BY 4.0)] 


\section{BACKGROUND}

Liver cirrhosis is the final common pathway for all chronic liver diseases. Cirrhosis currently causes 1.16 million deaths making it the 11th most common cause of death. Major cause for mortality in these patients is the complications of liver cirrhosis. ${ }^{1,2}$ Systemic inflammation has now been proposed to play a crucial role in the natural history of progressive liver damage. Cirrhosis associated immune dysfunction syndrome (CAID) is an entity characterised by combination of systemic inflammation and immune deficiency state. It has been described as a multifactorial process which may be secondary to an infectious or non-infectious stimulus. ${ }^{3,4}$ The systemic inflammation has been attributed with worsening of liver failure and poor outcome. Dysfunction of innate and adaptive immune system leading to increase in pro inflammatory cytokines is responsible for local as well as systemic injury in patients with cirrhosis. $5,6,7$

With overwhelming evidence of role of inflammatory markers in pathogenesis of liver disease, a number of surrogate serum markers have been studied as a prognostic guide for predicting outcome and improving management of decompensated liver disease. Neutrophil lymphocyte ratio is one such inexpensive and easily available marker of systemic inflammation. Neutrophil count helps in identifying ongoing inflammation and lymphocyte count represents immune regulatory pathway. ${ }^{8}$ Studies have shown NLR to be useful in predicting outcome and mortality in patients with viral hepatitis, hepatocellular carcinoma, liver transplantation and non-alcoholic fatty liver disease. ${ }^{9,10,11,12}$

Identification of infectious episodes and complications in cirrhosis are challenging due to lack of typical signs and symptoms. Further, delay in treating complications leads to significant morbidity as well as mortality in these patients ${ }^{13}$. In developing countries where resources are deficient, NLR is an ideal test for early detection of infection and inflammation. Increased NLR helps in early identification of infections and timely administration of antibiotics thus improving outcome of patient. ${ }^{14}$ Recently, NLR has also emerged as a predictor of mortality independent of MELD scores in patients with cirrhosis and with hepatocellular carcinoma, as well as in candidates on the liver transplantation list.15,16,17 However, studies have not been conducted to assess role of NLR in predicting complications, decompensation and short term mortality in cirrhosis. Hence, we conducted this study to correlate the association of neutrophil to Lymphocyte ratio with complications of cirrhosis and assess the utility of NLR as a predictor of complication.

\section{METHODS}

A prospective observational study was conducted in internal medicine department of a tertiary care centre, K.S. Hegde Hospital, Mangalore, India from January 2017 to June 2018. All patients diagnosed to have liver cirrhosis based on clinical features and ultrasonography, who were admitted in the hospital for management of cirrhosis and its complications, were included in the study. Inclusion Criteria for cirrhosis on sonography were coarse echotexture, nodular liver with or without free fluid, Splenomegaly and portal hypertension. Patients who had hepatocellular carcinoma, on steroid therapy and febrile illness like Dengue fever, Leptospirosis and Malaria were excluded from study.

The study was approved by ethics committee and informed consent was obtained from participants and detailed history with clinical findings were recorded in the proforma. Emphasis was given to evaluate for aetiology of cirrhosis. Patients with cirrhosis and without ascites or varices were considered as compensated cirrhosis and patients with cirrhosis and ascites, variceal bleed or encephalopathy were considered as decompensated cirrhosis. Laboratory tests were done for all patients which included complete blood count, renal function and liver function test. Ascitic fluid analysis was performed only when indicated. Neutrophil to lymphocyte ratio (NLR) was calculated by dividing the neutrophil count by the Lymphocyte count. Complications of cirrhosis which included oesophageal varices with bleeding, hepatic encephalopathy and spontaneous bacterial peritonitis were recorded even if the patient developed the complication during the hospital stay. Outcome of admission that is, death or fit for discharge was noted.

\section{Statistical Analysis}

Analysis was carried out by statistical software SPSS 20. Mean and standard deviation of Neutrophil to Lymphocyte ratio along with confidence interval was calculated. Occurrence of different complications in the patients was expressed by percentage. Logistic regression analysis was carried out to predict the complications with Neutrophil to lymphocyte ratio. To test for the association between categorical variables Chi square test was used value $<0.05$ was considered as statistically significant.

\section{RESULTS}

Our study enrolled 120 patients (108 male and 12 females) based on our diagnostic criteria. Mean age for the cohort was $53.91 \pm 10.9$ with maximum patients between the age group 51-60 (38.3\%). Alcoholic liver disease (96) was the commonest aetiology for the cirrhosis. A total of 34 (28.3\%) patients had cirrhosis with portal hypertension without evidence of oesophageal varices and were categorized a compensated cirrhosis. Among the decompensated Cirrhosis, 47 (39.2\%) patients had oesophageal varices with evidence of bleeding and $39(32.1 \%)$ patients had cirrhosis with hepatic encephalopathy and spontaneous bacterial peritonitis (SBP) as complications. Baseline demographic and laboratory characteristics of the population are shown in Table I.

Mean NLR for the patients was $5.824 \pm 5.632$ (95\% CI 4.816.84). Patients were divided into 5 groups on basis of NLR. distribution of NLR is demonstrated in Table II.

Twenty-one Patients (42\%) with NLR of 1-3 had compensated cirrhosis. It was observed that as the NLR increased, percentage of patients with decompensated cirrhosis also increased and 11 patients $(91.7 \%)$ with NLR $>12$ had decompensation. However, Chi- square test of association between NLR and decompensation was not statistically significant $(p=0.070)$. It was also noticed that as the NLR 
increased, the incidence of complications also increased with $66.7 \%$ of patients with NLR $>12$ having more than 2 complications that is hepatic encephalopathy and SBP (Table III). Chi- square test of association with likelihood ratio between NLR and complication was used, and $p$ value was found to be significant $(p=0.004)$. Five patients $(41.7 \%)$ with NLR $>12$ had mortality as the outcome. Chi square test of association with likelihood ratio between NLR and mortality was statistically significant $(\mathrm{p}=0.03)$.

\begin{tabular}{|c|c|}
\hline Characteristics & No. of Patients (\%) $(n=120)$ \\
\hline Gender (male/female) & $108 / 12$ \\
\hline Age (years) & $53.91 \pm 10.9$ \\
\hline \multicolumn{2}{|c|}{ Aetiology of Cirrhosis } \\
\hline Alcoholic liver disease & $96(80 \%)$ \\
\hline Cryptogenic & $13(10.8 \%)$ \\
\hline Non-alcoholic fatty liver disease & $5(4.1 \%)$ \\
\hline Wilson's disease & $4(3.33 \%)$ \\
\hline Hemochromatosis & $1(0.83 \%)$ \\
\hline Hepatitis B & $1(0.83 \%)$ \\
\hline \multicolumn{2}{|c|}{ State of Cirrhosis } \\
\hline Compensated & $34(28.3 \%)$ \\
\hline Decompensated & $86(71.7 \%)$ \\
\hline \multicolumn{2}{|c|}{ Complications } \\
\hline Portal hypertension without varices & $34(28.3 \%)$ \\
\hline Portal hypertension with variceal bleed & $47(39.2 \%)$ \\
\hline Hepatic encephalopathy and SBP & $39(32.5 \%)$ \\
\hline \multicolumn{2}{|l|}{ Outcome } \\
\hline Death & $13(10.8 \%)$ \\
\hline Alive & $106(88.33 \%)$ \\
\hline Left against Medical advice & $1(0.83 \%)$ \\
\hline Total Leucocyte count (cells/cumm) & $10787 \pm 7842$ \\
\hline NLR (mean \pm standard deviation) & $5.8250 \pm 5.632$ \\
\hline \multicolumn{2}{|c|}{ Table I. Baseline Characteristics of the Study Population } \\
\hline
\end{tabular}

\begin{tabular}{|ccc|}
\hline NLR & No. of Patients (n) & Percentage (\%) \\
\hline $1-3$ & 50 & 41.8 \\
$3.1-6$ & 35 & 29.2 \\
$6.1-9$ & 20 & 16.7 \\
$9.1-12$ & 3 & 2.5 \\
$>12$ & 12 & 10.0 \\
Total & $\mathbf{1 2 0}$ & $\mathbf{1 0 0 . 0}$ \\
\hline \multicolumn{3}{|c}{} \\
\hline \multicolumn{3}{c}{ Table II. Distribution of NLR } \\
\hline
\end{tabular}

\begin{tabular}{|c|c|c|c|c|c|c|c|c|}
\hline \multirow[b]{2}{*}{$\stackrel{9}{z}$} & \multicolumn{2}{|c|}{ State } & \multicolumn{2}{|c|}{ Outcome } & & \multicolumn{3}{|c|}{ Complications } \\
\hline & 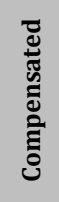 & 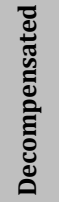 & 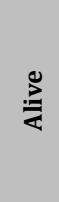 & 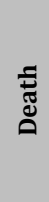 & 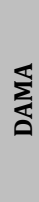 & 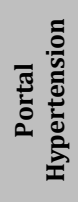 & 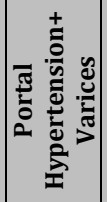 & 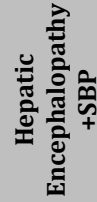 \\
\hline $1-3$ & 21 & 29 & 45 & 5 & 0 & 21 & 18 & 11 \\
\hline $3.1-6$ & 9 & 26 & 33 & 2 & 0 & 8 & 20 & 7 \\
\hline $6.1-9$ & 3 & 17 & 19 & 1 & 0 & 3 & 5 & 12 \\
\hline $9.1-12$ & 1 & 2 & 3 & 0 & 0 & 1 & 1 & 1 \\
\hline$>12$ & 1 & 11 & 6 & 5 & 1 & 1 & 3 & 8 \\
\hline $\mathrm{p}$ value & $\mathrm{pval}$ & 0.070 & $\mathrm{p} \mathrm{val}$ & $=0.03$ & & & value $=0.0 \mathrm{C}$ & \\
\hline
\end{tabular}

\begin{tabular}{|cccccc|}
\hline Variable & Coefficient & $\begin{array}{c}\text { Standard } \\
\text { Error }\end{array}$ & P-Value & $\begin{array}{c}\text { Odds } \\
\text { Ratio }\end{array}$ & $\begin{array}{c}\mathbf{9 5 \%} \\
\text { Confidence } \\
\text { Interval }\end{array}$ \\
NLR & 0.6606 & 0.3635 & 0.0691 & 1.9360 & $\begin{array}{c}0.9495- \\
3.9475\end{array}$ \\
Age & -0.0539 & 0.0222 & 0.0154 & 0.9476 & $\begin{array}{c}0.9072- \\
0.9898\end{array}$ \\
Sex & -1.2260 & 1.0744 & 0.2538 & 0.2935 & $\begin{array}{c}0.0357- \\
2.4105\end{array}$ \\
Aetiology & 0.6591 & 0.4958 & 0.1837 & 1.9331 & $\begin{array}{c}0.7316- \\
5.1080\end{array}$ \\
\hline
\end{tabular}

Table IV. Binary Logistic Regression Analysis NLR with Complications

\section{Logistic Regression Analysis}

Logistic regression analysis was done to assess ability of NLR to predict complications in patient with cirrhosis. For the purpose of logistic regression analysis patients were divided into 2 groups based on their complications. First group of patients consisted of cirrhotic patients with only portal hypertension and second group included cirrhotic patients with complications such as oesophageal varices with variceal bleed, hepatic encephalopathy and spontaneous bacterial peritonitis. In our study p value for NLR, age, sex and aetiology of cirrhosis was not significant, and it could not predict the complication in our study group.

\section{DISCUSSION}

In our study, we tried to examine the association of neutrophil to Lymphocyte ratio with complications, decompensation and short-term outcome of cirrhosis. We were able to demonstrate that increase in NLR is associated with increased complications and mortality in patients with cirrhosis.

Blood Leucocyte count has previously been used as a surrogate marker for sepsis. Many studies demonstrated leukocyte count as an independent predictor of short-term mortality in patients with acute on chronic liver failure.18 However, in patients with cirrhosis it is difficult to make a diagnosis of infection solely based on leucocyte count as it has its own drawbacks. Cirrhotic patients demonstrate abnormal haematological parameters which include anaemia, thrombocytopenia and leukopenia. The pathogenesis described for pancytopenia is multifactorial, with portal hypertension induced sequestration and bone marrow suppression accounting for majority of the cases. ${ }^{19}$ Once a patient develops infection the total count is bound to rise. However, there are evidence which say in $50 \%$ bacteraemia total count can be normal. ${ }^{20}$ Hence there is no consensus for a leucocyte cut-off point for diagnosing infection. In our study $36.7 \%(n=44)$ of patients had a total count between 40008000 cells/cumm. In a study by Jung Hyun Kwon, et al ${ }^{21}$ mean total count was 8971 cells/cumm while in our study we had a mean of 10787 cells/cumm. However, it was worth to observe that $28.3 \%$ of patients had a total count more than 12000 cells/cumm and $9.2 \%$ had total counts less than 4000 cells/cumm. There was no significant difference in mortality in the groups based on the total counts.

NLR has been considered an inexpensive biomarker to reflect inflammation in clinical conditions like Ischaemic Heart disease, Pneumonia and Cancer. Sharma K et al demonstrated that there a strong correlation between NLR and coronary artery disease (CAD) and Optimum cut-off of NLR in his study for diagnosis of CAD was $2.13 .{ }^{22} \mathrm{H}$. Shimada et al in his study on Neutrophil lymphocyte ratio in gastric cancer concluded that in patients with a high NLR, the 5-year survival was significantly worse than that of patients with a low NLR. ${ }^{23}$ Most studies have shown a higher NLR is associated with poor outcome and prognosis. However, there is no consensus for a definitive cut off value for NLR. Lee et al conducted a study to evaluate the sex- and age-specific reference values of NLR in normal population in Korea. They concluded that mean NLR across all ages in men and women was $1.63(0.76)$ and 1.66 (0.82), respectively and also commented that NLR is lower in Asian population compared to western cohorts. ${ }^{24}$ In our study majority of patients had a mean NLR of 5.8250. This value was much higher than the NLR demonstrated in other studies. This could be explained by the fact that our cohort included patients admitted in hospital for management of cirrhosis or 
its complications and did not represent follow up patients in outpatient department. In a study conducted Murat Biyik et al they demonstrated a mean NLR of 2.72 and concluded that the mortality rate was significantly higher (odds ratio was 8.9 ) in patients with an NLR higher than 2.72 compared with lower NLR values. 15

Complication of cirrhosis and decompensation is the major cause of mortality in cirrhotic patients. Previous studies have not studied the association between the complications and NLR. We noticed that as the NLR increased, the incidence of complications also increased with $66.7 \%$ of patients with NLR $>12$ having more than 2 complications that is hepatic encephalopathy with SBP. Chi- square test of association with likelihood ratio between NLR and complication was used, and $p$ value was found to be statistically significant $(p=0.003)$. We also tested for the Association between NLR and decompensation in cirrhosis. It was indeed astonishing to note that $91.7 \%$ patients with NLR more than 12 were in decompensated state during admission. However, test of association with $\mathrm{p}$ value was 0.057 which was not significant. However, when we did a logistic regression analysis on our data, we were unable to demonstrate NLR, Age and Sex as a predictor of complication.

NLR has been demonstrated as an independent marker for predicting mortality in patient with cirrhosis. Studies have shown that NLR is a predictor of mortality, independent of Child-Turcotte-Pugh and MELD, for patients with stable liver cirrhosis. It has also been able to predict mortality in low MELD score groups. ${ }^{10,15}$ In a study conducted by Jung Hyun Kwon et al, it was demonstrated that NLR was a useful predictor of 1-month survival, particularly in Child-Pugh class C patients ${ }^{18}$. In our study NLR had a positive correlation with hospital mortality. As the NLR increased the incidence of mortality also increased which was demonstrated by test of association with $\mathrm{p}$ value of 0.03 . Long term mortality after discharge of patients with higher NLR cannot be commented as patients were not followed up in our study.

We did face certain limitation in our study. As the duration of our study was short, sample size was limited. In Our study NLR was calculated only one time at admission. Serial monitoring of NLR would be necessary to diagnose complications during hospital stay. We did not compare MELD and CTP score with NLR as our study wanted to reflect on acute complication and hospital mortality. Despite of these limitation we had a few strengths. Ours is the only study which correlated the NLR with complications and decompensation in cirrhosis. We were able to demonstrate an association between the two, but we were not able to develop a cut-off value for NLR for our patients admitted with cirrhosis.

\section{CONCLUSIONS}

Raised Neutrophil to Lymphocyte Ratio is strongly associated with complication and short-term mortality in patients with cirrhosis. It can also be used as prognostic marker and can assist in early detection of decompensation in these patients. Further research is needed to develop reference values for NLR for diagnosing complication and mortality in patients with cirrhosis.

\section{Abbreviations}

CTP: Child-Turcotte-Pugh.

MELD score: Model for End-Stage Liver Disease score.

NLR: Neutrophil-to-Lymphocyte Ratio.

Financial or Other Competing Interests: None.

\section{REFERENCES}

[1] Asrani SK, Kamath PS. Natural history of cirrhosis. Curr Gastroenterol Rep 2013;15(2):308.

[2] Asrani SK, Devarbhavi H, Eaton J, et al. Burden of liver diseases in the world. J Hepatol 2019;70(1):151-71.

[3] Albillos A, Lario M, Álvarez-Mon M. Cirrhosis-associated immune dysfunction: distinctive features and clinical relevance. J Hepatol 2014;61(6):1385-96.

[4] Dirchwolf M, Ruf AE. Role of systemic inflammation in cirrhosis: from pathogenesis to prognosis. World J Hepatol 2015;7(16):1974-81.

[5] Sipeki N, Antal-Szalmas P, Lakatos PL, et al. Immune dysfunction in cirrhosis. World J Gastroenterol 2014;20(10):2564-77.

[6] Selmi C, Mackay IR, Gershwin ME. The immunological milieu of the liver. Semin Liver Dis 2007;27(2):129-39.

[7] Aoyama T, Paik YH, Seki E. Toll-like receptor signaling and liver fibrosis. Gastroenterol Res Pract 2010;2010:1-8.

[8] Tritto G, Bechlis Z, Stadlbauer V, et al. Evidence of neutrophil functional defect despite inflammation in stable cirrhosis. J Hepatol 2011;55(3):574-81.

[9] Gong J, Liang YL, Zhou W, et al. Prognostic value of neutrophil-to-lymphocyte ratio associated with prognosis in HBV-infected patients. J Med Virol 2018;90(4):730-5.

[10] Kalra A, Wedd JP, Bambha KM, et al. Neutrophil-tolymphocyte ratio correlates with proinflammatory neutrophils and predicts death in low model for end-stage liver disease patients with cirrhosis. Liver Transpl 2017;23(2):155-65.

[11] Yilmaz H, Yalcin KS, Namuslu M, et al. Neutrophillymphocyte ratio (NLR) could be better predictor than Creactive protein (CRP) for liver fibrosis in non-alcoholic steatohepatitis (NASH). Ann Clin Lab Sci 2015;45(3):27886.

[12] Lin BY, Zhou L, Geng L, et al. High neutrophil-lymphocyte ratio indicates poor prognosis for acute-on-chronic liver failure after liver transplantation. World J Gastroenterol 2015;21(11):3317-24.

[13] Arvaniti V, D'Amico G, Fede G, et al. Infections in patients with cirrhosis increase mortality four-fold and should be used in determining prognosis. Gastroenterology 2010;139(4):1246-56.

[14] Liu X, Shen Y, Wang H, et al. Prognostic significance of neutrophil-to-lymphocyte ratio in patients with sepsis: a prospective observational study. Mediators Inflamm 2016;2016:1-8

[15] Biyik M, Ucar R, Solak Y, et al. Blood neutrophil-tolymphocyte ratio independently predicts survival in patients with liver cirrhosis. Eur J Gastroenterol Hepatol 2013;25(4):435-41. 
[16] Gao F, Li X, Geng M, et al. Pretreatment neutrophillymphocyte ratio: an independent predictor of survival in patients with hepatocellular carcinoma. Medicine (Baltimore) 2015;94(11):e639.

[17] Limaye AR, Clark V, Soldevila-Pico C, et al. Neutrophillymphocyte ratio predicts overall and recurrence-free survival after liver transplantation for hepatocellular carcinoma. Hepatol Res 2013;43(7):757-64.

[18] Bajaj JS, O'Leary JG, Reddy KR, et al. Survival in infectionrelated acute-on-chronic liver failure is defined by extrahepatic organ failures. Hepatology 2014;60(1):2506.

[19] Qamar AA, Grace ND. Abnormal hematological indices in cirrhosis. Can J Gastroenterol 2009;23(6):441-5.

[20] Lowsby R, Gomes C, Jarman I, et al. Neutrophil to lymphocyte count ratio as an early indicator of blood stream infection in the emergency department. Emerg Med J 2015;32(7):531-4.
[21] Kwon JH, Jang JW, Kim YW, et al. The usefulness of Creactive protein and Neutrophil to lymphocyte ratio for predicting the outcome in hospitalized patients with liver cirrhosis. BMC Gastroenterology 2015;15:146.

[22] Sharma K, Patel AK, Shah KH, et al. Is Neutrophil-tolymphocyte ratio a predictor of coronary artery disease in western Indians? Int J Inflam 2017;2017:1-8.

[23] Shimada H, Takiguchi N, Kainuma O, et al. High preoperative neutrophil-lymphocyte ratio predicts poor survival in patients with gastric cancer. Gastric Cancer 2010;13(3):170-6.

[24] Lee JS, Kim NY, Na SH, et al. Reference values of neutrophil-lymphocyte ratio, lymphocyte-monocyte ratio, platelet-lymphocyte ratio, and mean platelet volume in healthy adults in South Korea. Medicine (Baltimore) 2018;97(26):e11138. 